Article

\title{
Functional Connectivity between the Resting-State Olfactory Network and the Hippocampus in Alzheimer's Disease
}

\author{
Jiaming Lu ${ }^{1,2}$, Nicole Testa ${ }^{1}$, Rebecca Jordan ${ }^{1}$, Rommy Elyan ${ }^{1}$, Sangam Kanekar ${ }^{1}$, Jianli Wang ${ }^{1}$, \\ Paul Eslinger ${ }^{1,3}$, Qing X. Yang ${ }^{1,4}$, Bing Zhang ${ }^{2}$ and Prasanna R. Karunanayaka ${ }^{1,5,6, *}$ \\ 1 Department of Radiology, The Pennsylvania State University College of Medicine, Hershey, PA 17033, USA; \\ b101230039@gmail.com (J.L.); nikkites58@gmail.com (N.T.); rjordan@pennstatehealth.psu.edu (R.J.); \\ relyan@pennstatehealth.psu.edu (R.E.); skanekar@pennstatehealth.psu.edu (S.K); \\ jwang2@pennstatehealth.psu.edu (J.W); peslinger@pennstatehealth.psu.edu (P.E.); \\ qyang@pennstatehealth.psu.edu (Q.X.Y.) \\ 2 Medical School of Nanjing University, Nanjing 210008, China; zhangbing_nanjing@vip.163.com \\ 3 Department of Neurology, The Pennsylvania State University College of Medicine, Hershey, PA 17033, USA \\ 4 Department of Neurosurgery, The Pennsylvania State University College of Medicine, \\ Hershey, PA 17033, USA \\ 5 Department of Neural and Behavioral Sciences, The Pennsylvania State University College of Medicine, \\ Hershey, PA 17033, USA \\ 6 Department of Public Health Sciences, The Pennsylvania State University College of Medicine, \\ Hershey, PA 17033, USA \\ * Correspondence: pkarunanayaka@pennstatehealth.psu.edu; Tel.: +1-717-531-6069; Fax: +1-717-531-8486
}

Received: 2 October 2019; Accepted: 18 November 2019; Published: 25 November 2019

\begin{abstract}
Olfactory impairment is associated with prodromal Alzheimer's disease (AD) and is a risk factor for the development of dementia. AD pathology is known to disrupt brain regions instrumental in olfactory information processing, such as the primary olfactory cortex (POC), the hippocampus, and other temporal lobe structures. This selective vulnerability suggests that the functional connectivity (FC) between the olfactory network (ON), consisting of the POC, insula and orbital frontal cortex (OFC) (Tobia et al., 2016), and the hippocampus may be impaired in early stage AD. Yet, the development trajectory of this potential FC impairment remains unclear. Here, we used resting-state functional magnetic resonance imaging (rs-fMRI) data from the Alzheimer's Disease Neuroimaging Initiative (ADNI) to investigate FC changes between the $\mathrm{ON}$ and hippocampus in four groups: aged-matched cognitively normal $(\mathrm{CN})$, early mild cognitive impairment (EMCI), late mild cognitive impairment (LMCI), and AD. FC was calculated using low frequency fMRI signal fluctuations in the ON and hippocampus (Tobia et al., 2016). We found that the FC between the ON and the right hippocampus became progressively disrupted across disease states, with significant differences between EMCI and LMCI groups. Additionally, there were no significant differences in gray matter hippocampal volumes between EMCI and LMCI groups. Lastly, the FC between the ON and hippocampus was significantly correlated with neuropsychological test scores, suggesting that it is related to cognition in a meaningful way. These findings provide the first in vivo evidence for the involvement of FC between the ON and hippocampus in AD pathology. Results suggest that functional connectivity (FC) between the olfactory network (ON) and hippocampus may be a sensitive marker for Alzheimer's disease (AD) progression, preceding gray matter volume loss.
\end{abstract}

Keywords: olfactory network; hippocampus; functional connectivity; resting state; Alzheimer's disease 


\section{Introduction}

Alzheimer's disease (AD) is marked by the insidious onset of episodic memory loss, but early clinical symptoms also include sensory (i.e., vision, hearing, and olfaction) and motor impairments [1-6]. Of particular interest are the well-documented olfactory deficits that often precede cognitive impairment $[1,7,8]$. Moreover, these early-stage olfactory deficits often predict the onset of dementia $[7,9-14]$. A post-mortem study demonstrated a significant correlation between patients with impaired odor identification and increased density of tangles in the entorhinal cortex and CA1/subiculum region of the hippocampus [15]. These findings allude to widespread cortical neuronal loss that severely compromises anatomical and functional connections within and between sensory and cognitive networks in $\mathrm{AD}$, namely olfaction and memory [1].

There is ample evidence suggesting that olfactory network $(\mathrm{ON})$ impairment is a potential biomarker for detecting the initial neuropathological processes observed in AD [16-18]. Emerging evidence suggests that these olfactory impairments may predict the onset of $A D$, amnestic mild cognitive impairment (aMCI), and the presence of amyloid- $\beta(\mathrm{A} \beta)$ and tau pathology in cognitively normal adults. However, very few AD studies have investigated olfactory deficits from a brain network perspective. This is a critical step in understanding the relationship between AD pathology and olfactory deficits. More specifically, a network approach may clarify if olfactory deficits, such as odor-identification deficits, are to be linked to damage in the primary and secondary olfactory structures, or if they reflect secondary effects of damage in higher-order cortical areas affected by AD.

Studies of resting-state (RS) functional connectivity (FC) in AD holds great promise in revealing how brain network dynamics are altered by neurodegeneration [19]. Several resting-state functional magnetic resonance imaging (rs-fMRI) studies have reported characteristic disruptions in functional networks prominent in AD pathology [20-22]. Most RS brain networks are intrinsically organized, but there is evidence to suggest that these networks are also influenced by task performance $[23,24]$. Furthermore, while FC assesses the integration of spontaneous and evoked brain activity across distant brain regions and networks, it is also thought to be shaped by the brains' structural connectivity $[25,26]$. The resting-state $\mathrm{ON}$ is functionally connected to the hippocampus, which anchors the episodic memory system (Tobia et al., 2016) [27]. Additionally, task fMRI studies have shown similar FC patterns with the hippocampus [28], a finding that is consistent with behavioral studies linking olfactory performance to memory function $[10,11,29]$.

It must be noted that whether olfactory deficits are due to a dysfunction in the central or peripheral olfactory nervous system remains unclear. For example, using task fMRI, Vasavada et al. (2017) suggested that olfactory deficits are most likely caused by degeneration in the central olfactory nervous system. Likewise, Zhang et al. (2018) showed that the number of mature olfactory sensory neurons in the olfactory epithelium is reduced in apolipoprotein E (ApoE)-deficient mice and suggested a link between the olfactory mucosa (peripheral) and the pathogeneses of AD.

The goal of this study was to investigate the resting-state functional connectivity (RS-FC) between the ON and hippocampus using the Alzheimer's Disease Neuroimaging Initiative (ADNI) data. We analyzed the ON FC in subjects who were categorized as age-matched cognitively normal (CN), early mild cognitive impairment (EMCI), late mild cognitive impairment (LMCI), and AD. In line with the disconnection hypothesis of $\mathrm{AD}$, widespread neuronal loss and brain network dysfunction were expected to cause olfactory impairments. Since olfactory deficits emerge early in the pathological cascade, we hypothesized that loss of functional coherence in olfactory structures may disrupt ON FC patterns [30-32]. Further, given that cognitive decline becomes progressively worse across the disease state, we expected a similar trajectory of impairment in the FC between the $\mathrm{ON}$ and the hippocampus. 


\section{Materials and Methods}

\subsection{Participants}

Resting-state fMRI data from 147 subjects (male, designated as "M") were obtained from the ADNI (http://www.adni-info.org/) dataset. Of the 147 subjects, 44 subjects were designated as CN (mean age $=74.18,17 \mathrm{M}), 46$ as EMCI (mean age $=71.69,19 \mathrm{M}), 31$ as LMCI (mean age $=72.41,18 \mathrm{M})$, and 26 as $\mathrm{AD}$ (mean age $=71.55,11 \mathrm{M}$ ) during the initial visit of the ADNI-GO or ADNI-2 phases (Table 1). EMCI and LMCI subjects were diagnosed based on the criteria described in the ADNI-2 procedure manual (http://www.adni-info.org/). Briefly, the criteria for diagnosing a subject with E/LMCI were as follows: (1) a subjective memory concern as reported by the subject, study partner, or clinician; (2) abnormal memory function documented by performance on the Logical Memory II subscale (Delayed Paragraph Recall, Paragraph A only) from the Wechsler Memory Scale-Revised (the maximum score is 25) based on the following education adjusted cutoffs-[a] 9 to 11 for sixteen or more years of education, [b] 5 to 9 for 8 to fifteen years of education, and [c] 3 to 6 for zero to seven years of education-(3) the Mini-Mental State Exam (MMSE) score is between 24 and 30 (inclusive); (4) if subjects with less than 8 years of education score outside of this inclusion range, exceptions may be made at the discretion of the project director; (5) Clinical Dementia Rating score of 0.5, with a Memory Box score of at least 0.5; and (6) general cognition and functional performance are sufficiently preserved such that a diagnosis of AD cannot be made by the site physician at the time of the screening visit. Additionally, all subjects were given the Alzheimer 's disease Assessment Scale cognitive subscale (ADAS-cog), Rey auditory verbal learning test (RAVLT), Montreal Cognitive Assessment (MoCA), and the Functional Activities Questionnaire (FAQ). All study subjects met the ADNI inclusion and exclusion criteria, which have been described previously [33] and can be found at http://www.adni-info.org/. Appropriate institutional review board approval was obtained at each ADNI site and informed consent was obtained from each subject or authorized representative.

Table 1. Demographics and neuropsychological data for all groups.

\begin{tabular}{cccccc}
\hline & CN $(\boldsymbol{n}=\mathbf{4 4})$ & EMCI $(\boldsymbol{n}=\mathbf{4 6})$ & LMCI $(\boldsymbol{n = 3 1 )}$ & AD $(\boldsymbol{n}=\mathbf{2 6})$ & $\boldsymbol{p} \#$ \\
\hline Male, No. $(\%)$ & $17(38.6)$ & $19(41.3)$ & $18(58.1)$ & $11(42.3)$ & 0.518 \\
Age & $74.18(6.1)$ & $71.69(7.3)$ & $72.41(7.4)$ & $71.55(7.3)$ & 0.37 \\
Education & $16.50(2.7)$ & $15.65(2.6)$ & $16.90(2.3)$ & $15.31(2.8)$ & 0.052 \\
MMSE & $28.86(1.4)$ & $28.39(1.6)$ & $27.74(1.6)$ & $22.54(2.6)$ & $<0.001$ \\
MoCA & $25.68(2.1)$ & $24.00(2.8)$ & $22.47(3.2)$ & $15.88(5.7)$ & $<0.001$ \\
CDR & $0.045(0.2)$ & $1.42(0.9)$ & $1.73(0.93)$ & $4.46(1.4)$ & $<0.001$ \\
RAVLT & $44.23(8.1)$ & $37.93(10.3)$ & $33.16(7.4)$ & $22.46(7.7)$ & $<0.001$ \\
ADAS11 & $5.69(2.1)$ & $7.93(3.4)$ & $11.09(4.7)$ & $23.19(8.2)$ & $<0.001$ \\
ADAS13 & $9.19(3.7)$ & $12.52(5.2)$ & $17.55(7.0)$ & $34.23(9.7)$ & $<0.001$ \\
FAQ & $0.11(0.5)$ & $2.48(3.9)$ & $4.871(4.9)$ & $15.038(7.4)$ & $<0.001$ \\
\hline
\end{tabular}

CN: Cognitively normal; EMCI: early mild cognitive impairment; LMCI: late mild cognitive impairment; AD: Alzheimer's disease; RAVLT: Rey auditory verbal learning test; CDR: clinical dementia rating; ADAS: Alzheimer's disease assessment scale; MMSE: Mini-Mental State Examination; MoCA: Montreal Cognitive Assessment; FAQ: Functional Activities Questionnaire; *: chi-square test; \#: Kruskal-Wallis ANOVA test.

\subsection{Image Preprocessing}

A detailed description of the resting-state fMRI and volumetric image acquisition protocols can be found at http://www.adni-info.org. The fMRI and volumetric data were processed with the Data Processing Assistant for Resting-State fMRI advanced edition (http://rfmri.org/DPARSFA) [34], which is based on statistical parametric mapping (http:/www.fil.ion.ucl.ac.uk/spm) and the toolbox for data processing and analysis of brain imaging (DPABI, http://rfmri.org/DPABI) [35]. Slice timing, head motion correction, and spatial normalization to the standard Montreal Neurological Institute (MNI) Echo Planar Imaging (EPI) template with a resolution of $3 \times 3 \times 3 \mathrm{~mm}^{3}$ were performed. As recommended by Anderson et al. (2011) and Murphy et al. (2009), the global signal regression 
was not performed to avoid introducing distortions into the time-series data [36,37]. All subjects in our study had a head movement of less than $3 \mathrm{~mm}$ translation and less than $3^{\circ}$ angular rotation in any direction (out of six) during fMRI scanning. Finally, fMRI data was detrended and band-pass filtered $(0.01-0.08 \mathrm{~Hz}$ ) before conducting the functional connectivity (FC) analysis. We also performed amplitude of low-frequency fluctuations (ALFF) and regional homogeneity (ReHo) analyses, using DPABI, to investigate the intensity and homogeneity of spontaneous brain activity in the primary olfactory cortex (POC) and hippocampus.

\subsection{Olfactory Network}

Based on published fMRI task activation studies, the olfactory network includes the POC, insula, and orbital frontal cortex (OFC) [27,38]. Seed time courses were extracted from preprocessed data in MNI space (( $x y z)$ coordinates) as the average time course within a five-voxel radius centered on coordinates defined from previous activation studies (see Supplementary Material Figure S1). Seeds comprising the core $\mathrm{ON}$ were obtained from a meta-analysis that identified these three bilateral brain regions as most likely to be activated by olfactory stimulation [38]. They included the piriform cortex ([-22 $0-14]$, [ $222-12])$, the OFC ([-24 $30-10]$, [28 $34-12])$, and the insula ([-30 18 6], [28 16 8]). Networks were computed as FC maps that survived a $p$ value of less than 0.01 with a minimum cluster size (k) of 60 voxels.

\subsection{Statistical Analysis}

Demographic (age and education) and neuropsychological data (MMSE, MoCA, CDR [Clinical Dementia Rating], RAVLT, ADAS11, ADAS13, FAQ) were compared using the Kruskal-Wallis ANOVA test. Pairwise group comparisons were performed using the Mann-Whitney $U$ test. The sex ratio between groups was compared using the chi-square test.

Whole brain and regions of interest (ROI) analyses, including group differences, were assessed using statistical inference performed at the voxel level with an FDR (False Discovery Rate) correction for multiple comparisons $(p<0.05)$ in DPABI and AFNI (https://afni.nimh.nih.gov/). Finally, an explorative correlation analysis was performed to test the significance of the relationship between FC values and the neuropsychological test scores described above.

\subsection{Volumetric Analysis}

A Bayesian model-based segmentation toolbox in the FMRIB Software Library (also known asFSL) (FIRST; http://fsl.fmrib.ox.ac.uk/fsl/fslwiki/FIRST) was used to segment each anatomical image and create vertex meshes for left and right hippocampus. Quality control of the subcortical segmentations was performed by an experienced image analyst, following FSL FIRST guidelines (https://fsl.fmrib.ox. ac.uk/fsl/fslwiki/FIRST/UserGuide). No participants were excluded because of poor segmentation of one or more structures. We computed the left and right hippocampus volumes by generating masks for both hippocampi in 3D volume space and multiplying the number of voxels in the mask by the voxel size.

\subsection{Relationship between ON FC and Neuropsychological Test Scores}

A Pearson correlation analysis was performed between the ON FC and neuropsychological test scores, including RAVLT, ADAS11, and ADAS13. The RAVLT is a list learning task that assess multiple aspects of verbal learning and memory. The ADAS11 and 13 are measures of global cognition, including memory, reasoning, language, orientation, ideational praxis, and constructional praxis. The test is scored in terms of errors, with higher scores reflecting poorer performances. Because this analysis was exploratory in nature, a statistical significance level of $p<0.05$ was used. 


\section{Results}

\subsection{Demographic and Neuropsychological Data}

Demographic and neuropsychological test score data are tabulated in Table 1. No significant differences in age, gender, and education level were found between groups. Significant differences were found between groups on neurospychological test scores $(p<0.001)$. Pairwise comparisons are tabulated in Table 2. As expected, neuropsychological test scores were significantly impaired in AD compared to the CN, EMCI, and LMCI groups.

Table 2. Pairwise comparisons between groups.

\begin{tabular}{ccccccc}
\hline & $\begin{array}{c}\text { CN } \\
\text { vs. EMCI }\end{array}$ & $\begin{array}{c}\text { CN } \\
\text { vs. LMCI }\end{array}$ & CN vs. AD & $\begin{array}{c}\text { EMCI } \\
\text { vs. LMCI }\end{array}$ & $\begin{array}{c}\text { EMCI } \\
\text { vs. AD }\end{array}$ & $\begin{array}{c}\text { LMCI } \\
\text { vs. AD }\end{array}$ \\
\hline & & & $p$ & & & \\
\hline Education & 0.095 & 0.632 & 0.048 & 0.046 & $<0.001$ & 0.024 \\
MMSE & 0.191 & 0.007 & $<0.001$ & 0.125 & $<0.001$ & $<0.001$ \\
MoCA & 0.018 & $<0.001$ & $<0.001$ & 0.053 & $<0.001$ & 0.001 \\
CDR & $<0.001$ & $<0.001$ & $<0.001$ & 0.344 & $<0.001$ & $<0.001$ \\
RAVLT & $<0.001$ & $<0.001$ & $<0.001$ & 0.058 & $<0.001$ & $<0.001$ \\
ADAS11 & 0.014 & $<0.001$ & $<0.001$ & 0.011 & $<0.001$ & $<0.001$ \\
ADAS13 & 0.017 & $<0.001$ & $<0.001$ & $<0.001$ & $<0.001$ & $<0.001$ \\
FAQ & $<0.001$ & $<0.001$ & $<0.001$ & 0.011 & $<0.001$ & $<0.001$ \\
\hline
\end{tabular}

$\mathrm{CN}$ : cognitively normal; EMCI: early mild cognitive impairment; LMCI: late mild cognitive impairment; AD: Alzheimer's disease; RAVLT: Rey auditory verbal learning test; CDR: clinical dementia rating; ADAS: Alzheimer's disease assessment scale; MMSE: Mini-Mental State Examination; MoCA: Montreal cognitive assessment; FAQ: Functional Activities Questionnaire. Pairwise comparisons were performed using the Mann-Whitney U test.

\subsection{ON FC Differences and the Region of Interest (ROI) Analysis}

We conducted a whole brain one-way ANOVA and detected ON FC group differences in the right hippocampus, with no significant differences in the left hippocampus (Figure 1). The coordinate of the peak value voxel in the right hippocampus is tabulated in Table 3. A subsequent region of interest (ROI) analysis of the ON FC is shown in Figure 2. Of note, the ON FC is significantly different between EMCI and LMCI groups.

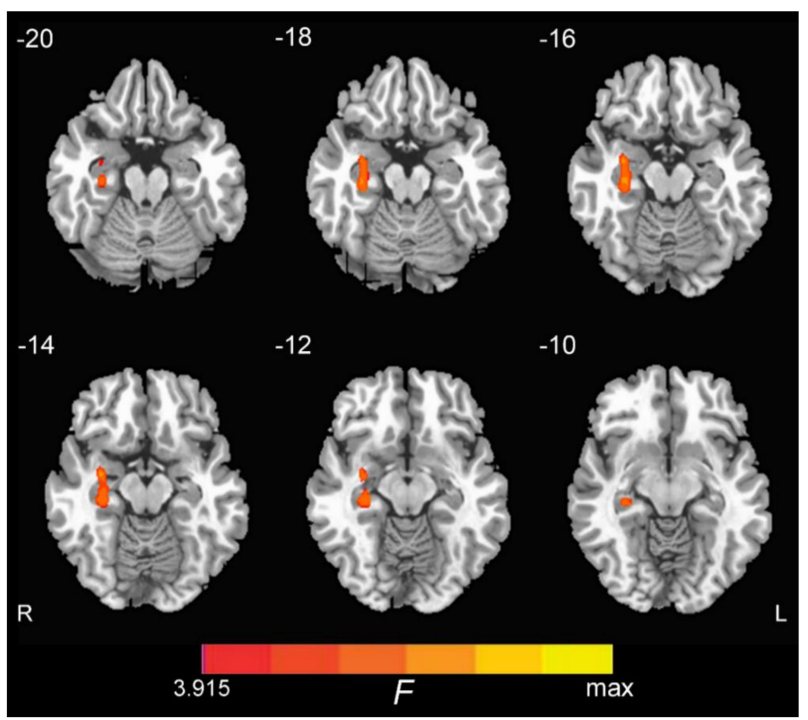

Figure 1. Group differences among CN (cognitively normal), EMCI (early mild cognitive impairment), LMCI (late mild cognitive impairment), and AD (Alzheimer's disease) groups of the olfactory network (ON) functional connectivity (FC) to the right hippocampus ( $p<0.01$, AlphaSim corrected). 
Table 3. Coordinates of the peak voxels in the hippocampus.

\begin{tabular}{cccccc}
\hline Cluster & $\begin{array}{c}\text { Cluster Size } \\
\text { (Voxel) }\end{array}$ & \multicolumn{3}{c}{ MNI Coordinates } & $\boldsymbol{t}$ Value \\
\cline { 3 - 6 } & 74 & 33 & -6 & -15 & 7.59 \\
\hline $\begin{array}{c}\text { Right } \\
\text { Hippocampus }\end{array}$ & 74 & & $\boldsymbol{x}$ & & $\boldsymbol{z}$ \\
\hline
\end{tabular}

MNI: Montreal Neurological Institute.

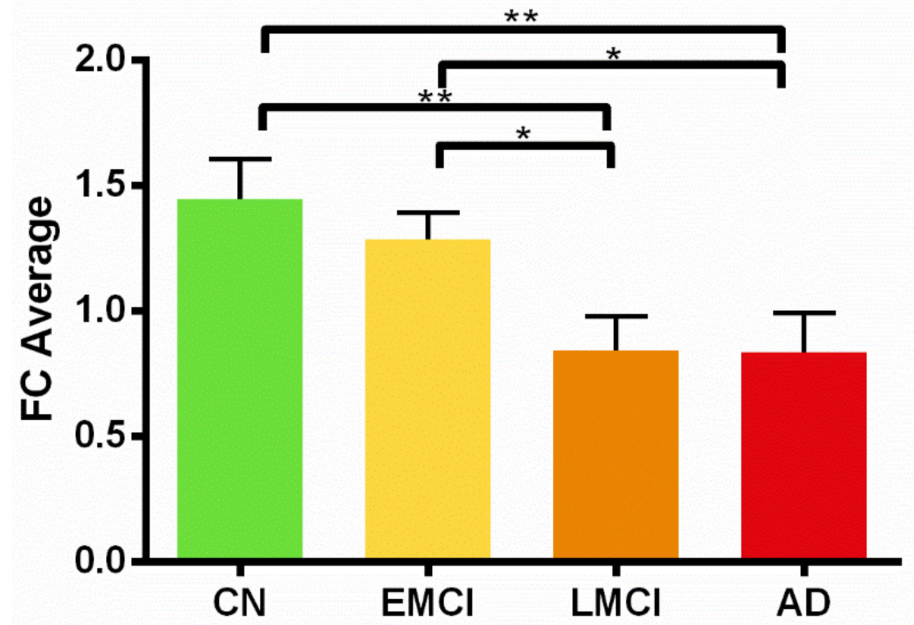

Figure 2. Differences in $\mathrm{ON} F C$ to the right hippocampus in $\mathrm{CN}, \mathrm{EMCI}, \mathrm{LMCI}$, and AD groups. *: $p<0.05 ;{ }^{* *}: p<0.01$. CN: cognitively normal; EMCI: early mild cognitive impairment; LMCI: late mild cognitive impairment; AD: Alzheimer's disease. Of note, the ON FC to the left hippocampus was not significantly different between groups.

\subsection{Region of Interest (ROI) Analysis of the Hippocampal Volume}

The hippocampal volume analysis by FSL revealed significant differences in the right hippocampal volume between the AD group and all other groups $(p<0.05$ FWE [Family-wise Error Rate] corrected for multiple comparisons). No significant differences in hippocampal volume were observed between the EMCI and LMCI (Figure 3). A similar pattern was observed for the left hippocampal volume (see Supplementary Material Figure S2). No correlations between the ON FC and hippocampal volume were significant in any group.

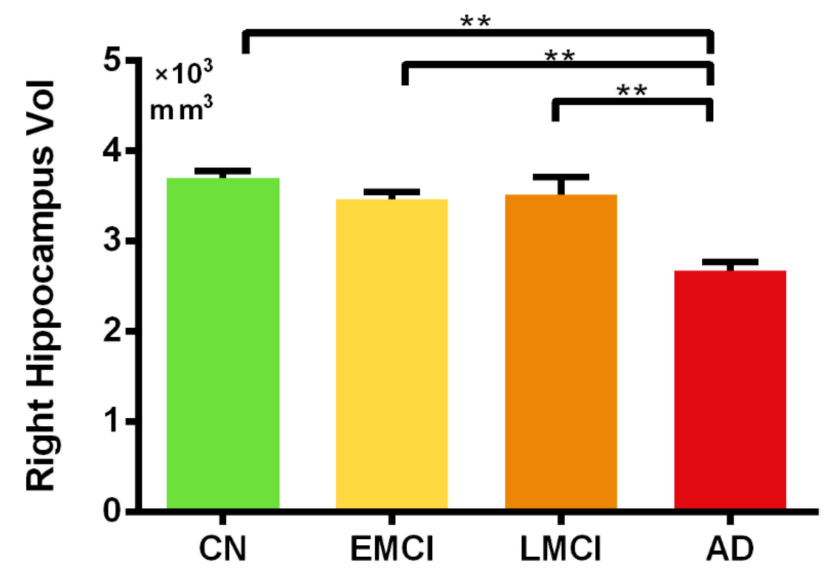

Figure 3. Differences in right hippocampal volume (vol) in the CN, EMCI, LMCI, and AD groups. *: $p<0.05 ;{ }^{* *}: p<0.01$. CN: cognitively normal; EMCI: early mild cognitive impairment; LMCI: late mild cognitive impairment; AD: Alzheimer's disease. 


\subsection{Correlation between ON FC and Neuropsychological Test Scores}

There was a significant positive correlation between the ON FC and RAVLT-immediate $(r=0.225$, $p=0.0083)$. Conversely, ON FC was negatively correlated with CDR $(\mathrm{r}=-0.178, p=0.038)$, ADAS11 $(\mathrm{r}=-0.195, p=0.018)$, and ADAS13 $(\mathrm{r}=-0.193, p=0.019)$. These exploratory correlation analyses were not corrected for multiple comparisons (Figure 4).
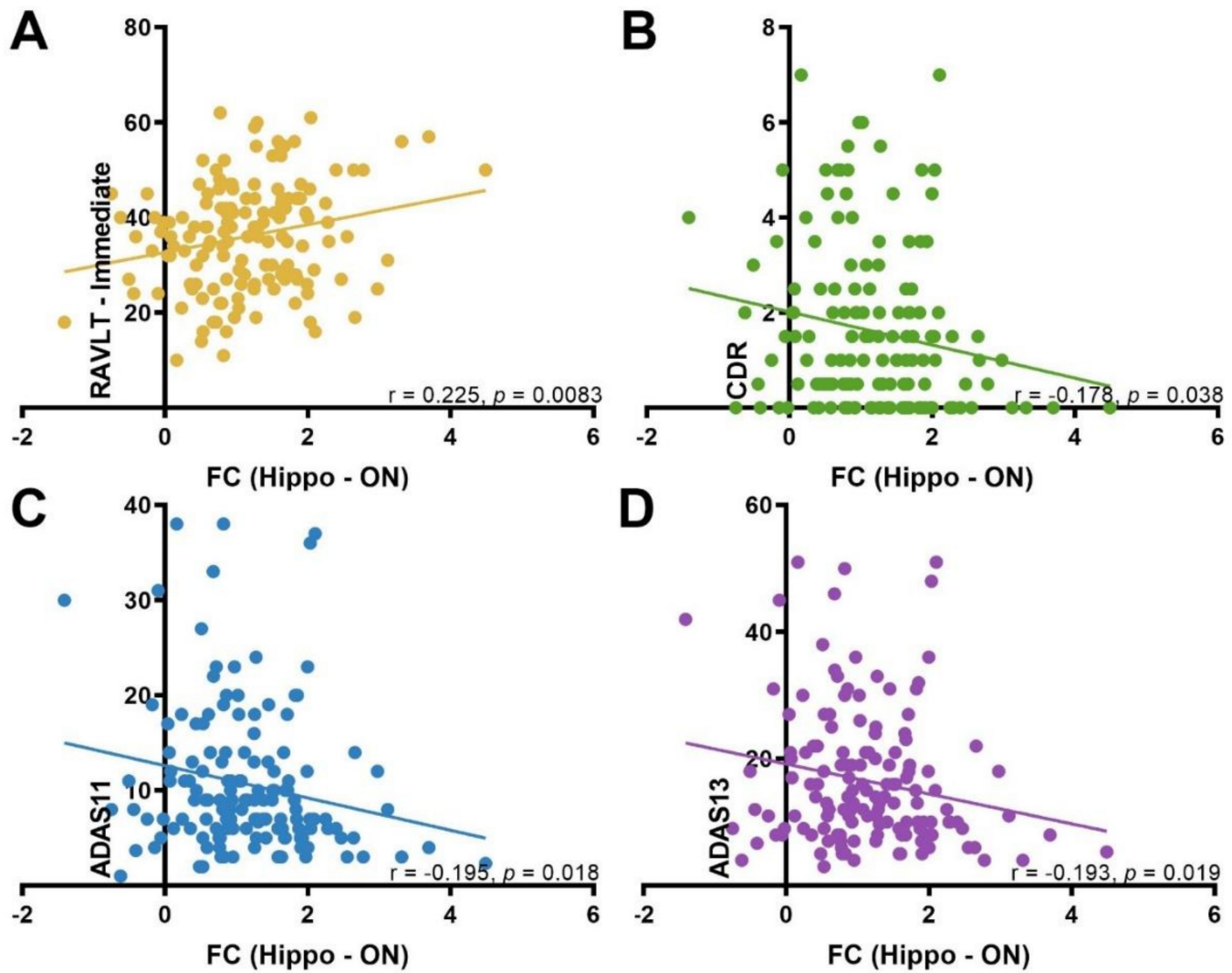

Figure 4. The correlations between each cognitive test score and the ON FC with the hippocampus. (A) RAVLT: Rey auditory verbal learning test; (B) CDR: clinical dementia rating; (C and D) ADAS: Alzheimer's disease assessment scale. FC: functional connectivity.

\section{Discussion}

The pathological cascade of AD suggested by Jack et al. (2010) highlighted the early emergence of olfactory impairments in AD. In fact, the level of olfactory impairment has demonstrated the ability to distinguish between disease stages and predict progression from MCI to AD [39]. Additionally, it has been suggested that early olfactory impairment may reflect the onset of AD, amnestic mild cognitive impairment (aMCI), and the presence (or formation) of amyloid- $\beta(\mathrm{A} \beta)$ and tau pathology in cognitively normal adults [1,40]. Furthermore, neurofibrillary tangles (NFTs) in AD are known to selectively disturb specific cortical layers in the hippocampal formation [41]. In turn, this may disrupt hippocampal projections [42] effectively isolating the hippocampus from the rest of the brain. This, combined with our finding of reduced ON FC to the hippocampus, may provide a basis for explanation of olfactory deficits in AD, corroborating the disconnection hypothesis [43]. Four main findings were generated in this study: first, the ON FC to the right hippocampus, which reflects the coherence of brain activity between the $\mathrm{ON}$ and the hippocampus, decreased depending on the AD disease state; second, the ON FC to the hippocampus was significantly different between the EMCI and LMCI groups; third, the ON FC to the hippocampus was a more sensitive indicator of AD progression compared to hippocampal volume in the early stages; and fourth, ON FC was meaningfully related to cognitive functions, based on significant correlation with auditory verbal learning scores (RAVLT). 
Thus, FC measures of the ON may offer unique opportunities to investigate direct and specific effects of local neurodegeneration to network disruption and functional deficits in AD.

Degeneration of the entorhinal cortex (ERC), part of the primary olfactory cortex (POC), affects activity in the hippocampus that memory processes (including odor-related) depend on. Neuroanatomically, an impaired entorhinal cortex will disrupt projections of the hippocampus that are necessary for successful memory formation [44-46]. Therefore, damage to the ERC may disconnect the hippocampus from the cerebral cortex $[47,48]$, a proposition supported by our results of reduced FC between the $\mathrm{ON}$ and hippocampus. However, it should be noted that our functionally-defined ROI did not include the ERC. Nevertheless, there is support to suggest that FC is a potential marker for memory decline in the early stages of $\mathrm{AD}[20,49]$. We further investigated hippocampus connectivity using the measure degree of centrality (DC), which quantifies direct connections of a given voxel with the rest of the brain [50]. As shown in Figure S3 of the Supplementary Material, we observed decreasing DC values in the right hippocampus, dependent on $\mathrm{AD}$ disease state. These results demonstrate potential isolation of the hippocampus from the rest of the brain in AD subjects [51].

Seed-based FC studies of the hippocampus have found widespread connectivity impairments in AD $[19,20,52]$. Using similar methodology, Li et al. (2002) and Greicius et al. (2004) showed reduced synchrony of low-frequency fluctuations (LFFs) and resting-state activity in the hippocampus of patients with AD. More specifically, Wang et al. (2006) noted a decrease in the right hippocampal connectivity to the medial prefrontal cortex (MPFC), ventral anterior cingulate cortex (VACC), and posterior cingulate cortex (PCC) in AD, likely indicating decreased activity of the default mode network (DMN) and contributing to episodic memory impairment. However, the left vs. right hippocampal involvement in olfaction and AD remains unclear and is an interesting topic for future research.

Furthermore, a recent olfactory fMRI paper by Karunanayaka et al. (2019) showed reduced task related $\mathrm{ON}$ activation and DMN suppression in mild cognitive impairment (MCI) and AD subjects compared to age-matched cognitively normal subjects. Together, these studies are consistent with the notion that decreased connectivity in the DMN and other networks are pervasive across broad brain regions in subjects with AD [53]. Combined with the current findings, a likely contributor of AD-related olfactory deficits is the disruption of ON connectivity to the hippocampus which is linked to the DMN [54]. Previously, we proposed a mesoscale brain network model that anatomically and functionally linked the DMN to the ON via the hippocampus [55]. That model, supported by the findings of the current study (Figure 5), may help differentiate patterns of olfactory deficits and their development in $\mathrm{AD}$ progression, leading to new studies of $\mathrm{AD}$ pathophysiology focusing on coupling impairments affecting network dynamics [56].

Our study is consistent with findings of decreased connectivity in brain networks in subjects across various AD disease states. While ON FC and hippocampal volume were not correlated in any group, we observed FC differences between EMCI and LMCI subjects in the absence of significant hippocampal volume differences. Interestingly, no differences in FC were observed between LMCI and AD subjects, which did have significant hippocampal volume differences. Based on these observations, our results suggest that hippocampal volume and ON FC may provide complementary information, with the latter being a more sensitive marker for AD progression, preceding volumetric loss.

We also investigated the POC volume using methods described in the Supplementary Materials. Although Figure S4 shows a decreasing trend in POC volume dependent on AD disease state, it did not reach significance. Unlike the hippocampus, the FSL software is unable to perform automatic segmentation of the POC. Thus, we used nonlinear transformations to project the POC in the standard space onto individual subject space before calculating POC volumes. This method may have introduced significant error into our POC volumetric analysis given the close proximity to air tissue boundaries in the brain. As a result, $\mathrm{POC}$ volume sensitivity in $\mathrm{AD}$ was compromised compared to hippocampal volume.

Unlike previous resting-state studies, the current study did not find increased FC between the ON and hippocampus in AD. Further, the ALFF and ReHo analyses in the POC and hippocampus 
did not detect any group differences. Since the ADNI data we analyzed did not include olfactory behavioral data, it is not possible to comment on compensatory connectivity in the current analysis. However, previous studies have hypothesized that increased activity and connectivity represent compensatory activity as cognition becomes impaired [57-60]. Alternatively, animal and human studies have suggested that increased activity may, in fact, reflect ongoing damage due to AD [61,62]. The latter hypothesis is congruent with our results, as demonstrated by widespread neuronal loss affecting loss of functional coherence in olfactory brain structures, leading to decreased FC between the $\mathrm{ON}$ and the hippocampus.

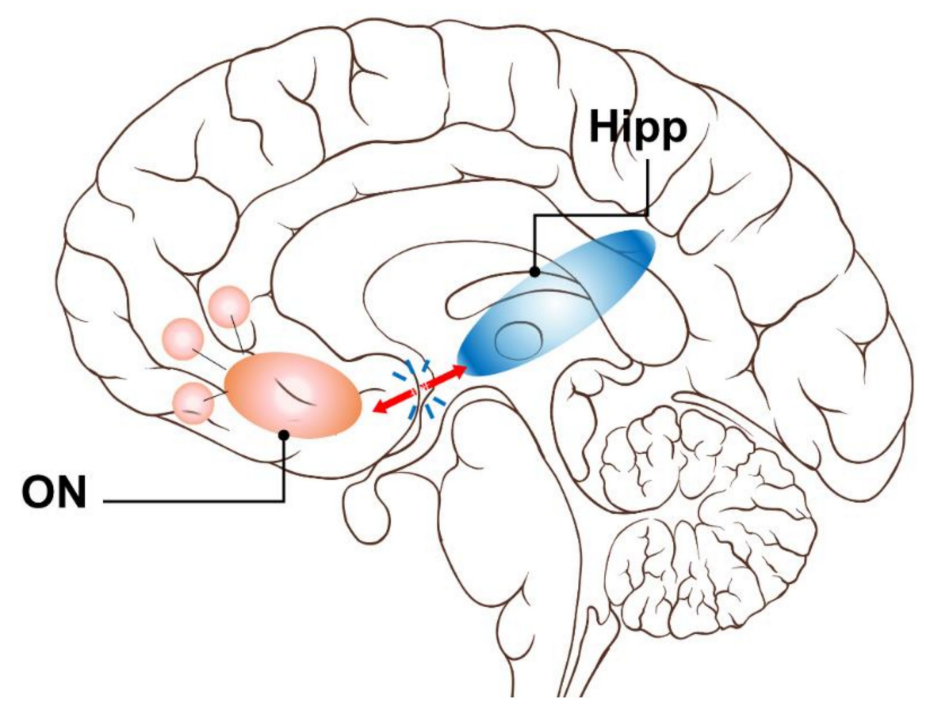

Figure 5. An FC model for olfactory deficits in AD. Early AD pathology compromises the ON FC to the hippocampus causing deficits in olfaction and memory. This Resting state fMRI, or rs-fMRI, model corroborates the model proposed by Lu et al. (2019) using olfactory task fMRI data. Based on the results of the current study, it is possible that the impaired functional connectivity between the ON and DMN in AD may be due to weakened or impaired ON hippocampus connectivity. Critically, this rs-fMRI model provides a testable hypothesis to relate AD neurodegeneration-to-olfactory impairment. In line with the brain network perspective, the proposed model provides pathophysiologic insight into neurodegenerative processes that may link olfaction to memory and other cognitive function deficits.

Our exploratory analysis indicated significant correlations between ON FC and neuropsychological test scores. Functional connectivity between the $\mathrm{ON}$ and the hippocampus was positively correlated with RAVLT, a measure of verbal memory. Postuma et al. (2011) reported a significant correlation between episodic verbal memory and olfactory impairment [63], a result that was reflected in our finding. Additionally, FC between the $\mathrm{ON}$ and the hippocampus was negatively correlated with ADAS scores. Given that lower scores reflect better performance, our results suggest that intact FC is related to better performance on a measure of global cognition. Lastly, intact ON FC was significantly related to lower clinician rating on the CDR, which is a measure of disease severity and categorization. However, it should be noted that these correlations must be interpreted with caution when applied to the general population.

These findings may help establish specific relationships between ON FC and pathological changes in the POC, hippocampus, and AD-related behavioral measures. Such relationships have been previously hypothesized based on postmortem studies where severe AD pathology has been found in olfactory structures. The current results provide additional in vivo evidence to support the involvement of the ON beyond the olfactory bulb and tract in AD pathology. This gives credence to the possibility that $\mathrm{ON}$ connectivity could serve as a possible predictor of cognitive decline in AD. 
Limitations of the Study

Although the ON FC results survived statistical correction for multiple comparisons, our findings should be considered preliminary in the absence of clinical olfactory testing (e.g., University of Pennsylvania Smell Identification Test (UPSIT). Additionally, the ADNI data set used lacks control for potential confounding factors associated with olfactory function (e.g., nasal pathology). As such, our exploratory correlation analyses need to be replicated with the inclusion of potential confounding factors. We did not find differences in ON FC with respect to APOE4 status, in any group. Future studies should focus on investigating the relevance of olfactory testing and its applicability to those with genetic risk factors for the development of AD. Other limitations include the cross sectional nature of this data set and the absence of longitudinal RS-fMRI data, olfactory data, genetic data, and neuropsychological data. These drawbacks underscore the need for follow-up studies, with a larger cohort and a more robust data collection protocol, to better elucidate ON FC as an indicator of olfactory performance and disease state in AD progression. Nevertheless, we hypothesize that the observed reduction in FC between the $\mathrm{ON}$ and the hippocampus in $\mathrm{AD}$ and MCI groups may constitute a significant risk factor for progressive decline and, ultimately, dementia. Based on the current results, this hypothesis is necessarily speculative.

The current study included subjects with motion parameters less than $3 \mathrm{~mm}$ translation and $3^{\circ}$ angular rotation in the 6 directions (using the standard motion correction procedure as implemented in Statistical Parametric Mapping 12, aka SPM 12). Therefore, given the number of subjects in the current study (121) and the focus on FC, we feel confident that we have addressed subject motion adequately in the current analysis. However, a better option would have been the framewise displacement method described in Power et al. (2019) that does address the effects of transient subject movements. This method is known to be superior at detecting spurious but systematic correlations in FC networks due to subject motion [64].

As mentioned in the introduction, evidence suggests that the number of mature olfactory sensory neurons in the olfactory epithelium is reduced in AD [65]. Therefore, future AD studies should focus on investigating the relationship between the reduced number of olfactory neurons at the periphery and the systems-level FC investigated in the current study. Studies of this nature may help delineate peripheral vs. central factors contributing to AD-related olfactory impairment. Lastly, with the expansion of knowledge related to AD pathophysiology, there are multiple ways to subtype AD patients (i.e., according to different genetic backgrounds and/or clinical presentations). Our study, however, focused on $\mathrm{AD}$ with memory deficits, one of the first clinical subtypes described [66]. Therefore, future studies are warranted to understand the relevance of resting-state functional connectivity differences in $A D$ subtypes other than amnestic MCI.

\section{Summary and Conclusions}

This research provided new knowledge of the neural substrates of resting-state FC between the $\mathrm{ON}$ and the hippocampus and its relationship to AD. Results indicated that the loss of hippocampal tissue volume lags behind disruption of resting-state FC between the $\mathrm{ON}$ and the hippocampus. Importantly, the ON was not preferentially affected in later stages of $\mathrm{AD}$, but was significantly involved in the early stages, which makes olfaction a potential sensitive indicator for $\mathrm{AD}$ progression. Based on the observed progressive decline of ON FC, our results lend support to the scientific premise that olfactory dysfunction in AD precedes the development of dementia. The results provide a strong foundation for future AD studies that focus on how prominent olfactory deficits in AD are related to neurodegeneration in the $\mathrm{ON}$ and hippocampus. Future research should also investigate if olfactory deficits signal progressive disruptions to the $\mathrm{ON}$ and its connectivity to the hippocampus. Olfactory deficits in prodromal AD patients may create a unique opportunity for rs-fMRI to directly address the functional consequences of observed neuropathological changes. In summary, our study provided in vivo rs-fMRI data showing functional connectivity degeneration between the $\mathrm{ON}$ and hippocampus in MCI, which provides some of the structural basis for the olfactory deficits in these 
patients. Specifically, significant differences in ON FC to right hippocampus were observed between EMCI and LMCI, highlighting the ability to differentiate between disease status. Importantly, ON-based FC could be used in conjunction with volumetric measurements of the POC and hippocampus and behavioral olfactory testing (e.g., UPSIT) to increase the diagnostic sensitivity and specificity of MCI patients. This development could contribute to an inexpensive, noninvasive predictor for cognitive decline in the early stages of $\mathrm{AD}[67]$.

Supplementary Materials: The following are available online at http://www.mdpi.com/2076-3425/9/12/338/s1, Figure S1: Olfactory network (ON), Figure S2: Left hippocampal volume in CN, EMCI, LMCI and AD groups., Figure S3: DC values in the right hippocampus in CN, EMCI, LMCI and AD groups., Figure S4: POC volume in $\mathrm{CN}, \mathrm{EMCI}, \mathrm{LMCI}$ and AD groups.

Author Contributions: Conceptualization, J.L. and P.R.K.; methodology, J.L. and P.R.K.; software, J.L., P.R.K., J.W., and Q.X.Y.; validation, J.L., P.R.K., and N.T.; formal analysis, N.T. and J.L.; investigation, P.R.K. and J.L.; resources, P.R.K. and B.Z.; data curation, N.T. and J.L.; writing-original draft preparation, R.J., R.E., P.R.K., and J.L.; writing - review and editing, S.K., P.E., R.E., and R.J.; visualization, N.T., R.E., and J.L.; supervision, P.R.K., P.E., S.K., B.Z., and Q.X.Y.; project administration, P.R.K. and, B.Z.; funding acquisition, P.R.K. and B.Z.

Funding: This research was funded by the Leader Family Foundation, a grant from the U.S. National Institute of Aging (R01-AG027771), National Institute of Neurological Disorders and Stroke (R01-NS099630), Pennsylvania Department of Health using Tobacco CURE Funds and the Department of Radiology, Penn State College of Medicine.

Conflicts of Interest: The authors declare no conflicts of interest.

\section{References}

1. Murphy, C. Olfactory and other sensory impairments in Alzheimer disease. Nat. Rev. Neurol. 2019, 15, 11-24. [CrossRef] [PubMed]

2. Albers, M.W.; Gilmore, G.C.; Kaye, J.; Murphy, C.; Wingfield, A.; Bennett, D.A.; Boxer, A.L.; Buchman, A.S.; Cruickshanks, K.J.; Devanand, D.P.; et al. At the interface of sensory and motor dysfunctions and Alzheimer's disease. Alzheimers Dement. 2015, 11, 70-98. [CrossRef] [PubMed]

3. Fischer, M.E.; Cruickshanks, K.J.; Schubert, C.R.; Pinto, A.A.; Carlsson, C.M.; Klein, B.E.; Klein, R.; Tweed, T.S. Age-Related Sensory Impairments and Risk of Cognitive Impairment. J. Am. Geriatr. Soc. 2016, 64, 1981-1987. [CrossRef] [PubMed]

4. Davies, H.R.; Cadar, D.; Herbert, A.; Orrell, M.; Steptoe, A. Hearing Impairment and Incident Dementia: Findings from the English Longitudinal Study of Ageing. J. Am. Geriatr. Soc. 2017, 65, 2074-2081. [CrossRef]

5. Deal, J.A.; Betz, J.; Yaffe, K.; Harris, T.; Purchase-Helzner, E.; Satterfield, S.; Pratt, S.; Govil, N.; Simonsick, E.M.; Lin, F.R. Hearing Impairment and Incident Dementia and Cognitive Decline in Older Adults: The Health ABC Study. J. Gerontol. A Biol. Sci. Med. Sci. 2017, 72, 703-709. [CrossRef]

6. Hyman, B.T. The neuropathological diagnosis of Alzheimer's disease: Clinical-pathological studies. Neurobiol. Aging 1997, 18 (Suppl. 4), S27-S32. [CrossRef]

7. Waldton, S. Clinical observations of impaired cranial nerve function in senile dementia. Acta Psychiatr. Scand. 1974, 50, 539-547. [CrossRef]

8. Doty, R.L.; Reyes, P.F.; Gregor, T. Presence of both odor identification and detection deficits in Alzheimer's disease. Brain Res. Bull. 1987, 18, 597-600. [CrossRef]

9. Ferreyra-Moyano, H.; Barragan, E. The olfactory system and Alzheimer's disease. Int. J. Neurosci. 1989, 49, 157-197. [CrossRef]

10. Knupfer, L.; Spiegel, R. Differences in olfactory test performance between normal aged, Alzheimer and vascular type dementia individuals. Int. J. Geriatr. Psychiatry 1986, 1, 3-14. [CrossRef]

11. Murphy, C.; Gilmore, M.M.; Seery, C.S.; Salmon, D.P.; Lasker, B.R. Olfactory thresholds are associated with degree of dementia in Alzheimer's disease. Neurobiol. Aging 1990, 11, 465-469. [CrossRef]

12. Roberts, R.O.; Christianson, T.J.; Kremers, W.K.; Mielke, M.M.; Machulda, M.M.; Vassilaki, M.; Alhurani, R.E.; Geda, Y.E.; Knopman, D.S.; Petersen, R.C. Association between olfactory dysfunction and amnestic mild cognitive impairment and Alzheimer disease dementia. JAMA Neurol. 2016, 73, 93-101. [CrossRef] [PubMed] 
13. Roalf, D.R.; Moberg, M.J.; Turetsky, B.I.; Brennan, L.; Kabadi, S.; Wolk, D.A.; Moberg, P.J. A quantitative meta-analysis of olfactory dysfunction in mild cognitive impairment. A quantitative meta-analysis of olfactory dysfunction in mild cognitive impairment. J. Neurol. Neurosurg. Psychiatry 2017, 88, 226-232. [CrossRef] [PubMed]

14. Rahayel, S.; Frasnelli, J.; Joubert, S. The effect of Alzheimer's disease and Parkinson's disease on olfaction: A meta-analysis. Behav. Brain Res. 2012, 231, 60-74. [CrossRef]

15. Wilson, R.S.; Arnold, S.E.; Schneider, J.A.; Tang, Y.; Bennett, D.A. The relationship between cerebral Alzheimer's disease pathology and odour identification in old age. J. Neurol. Neurosurg. Psychiatry 2007, 78, 30-35. [CrossRef]

16. Braak, H.; Braak, E. Evolution of the neuropathology of Alzheimer's disease. Acta Neurol. Scand. Suppl. 1996, 165, 3-12. [CrossRef]

17. Braak, H.; Braak, E. Evolution of neuronal changes in the course of Alzheimer's disease. J. Neural. Transm. Suppl. 1998, 53, 127-140.

18. Braak, H.; Thal, D.R.; Ghebremedhin, E.; Del Tredici, K. Stages of the pathologic process in Alzheimer disease: Age categories from 1 to 100 years. J. Neuropathol. Exp. Neurol. 2011, 70, 960-969. [CrossRef]

19. Sheline, Y.I.; Raichle, M.E. Resting state functional connectivity in preclinical Alzheimer's disease. Biol. Psychiatry 2013, 74, 340-347. [CrossRef]

20. Wang, L.; Zang, Y.; He, Y.; Liang, M.; Zhang, X.; Tian, L.; Wu, T.; Jiang, T.; Li, K. Changes in hippocampal connectivity in the early stages of Alzheimer's disease: Evidence from resting state fMRI. Neuroimage 2006, 31, 496-504. [CrossRef]

21. Greicius, M.D.; Srivastava, G.; Reiss, A.L.; Menon, V. Default-mode network activity distinguishes Alzheimer's disease from healthy aging: Evidence from functional MRI. Proc. Natl. Acad. Sci. USA 2004, 101, 4637-4642. [CrossRef] [PubMed]

22. Li, S.J.; Li, Z.; Wu, G.; Zhang, M.J.; Franczak, M.; Antuono, P.G. Alzheimer Disease: Evaluation of a functional MR imaging index as a marker. Radiology 2002, 225, 253-259. [CrossRef] [PubMed]

23. Deco, G.; Jirsa, V.K.; McIntosh, A.R. Emerging concepts for the dynamical organization of resting-state activity in the brain. Nat. Rev. Neurosci. 2011, 12, 43-56. [CrossRef] [PubMed]

24. Cole, M.W.; Bassett, D.S.; Power, J.D.; Braver, T.S.; Petersen, S.E. Intrinsic and task-evoked network architectures of the human brain. Neuron 2014, 83, 238-251. [CrossRef] [PubMed]

25. Adachi, Y.; Osada, T.; Sporns, O.; Watanabe, T.; Matsui, T.; Miyamoto, K.; Miyashita, Y. Functional connectivity between anatomically unconnected areas is shaped by collective network-level effects in the macaque cortex. Cereb. Cortex 2012, 22, 1586-1592. [CrossRef]

26. Goni, J.; van den Heuvel, M.P.; Avena-Koenigsberger, A.; Velez de Mendizabal, N.; Betzel, R.F.; Griffa, A.; Hagmann, P.; Corominas-Murtra, B.; Thiran, J.P.; Sporns, O. Resting-brain functional connectivity predicted by analytic measures of network communication. Proc. Natl. Acad. Sci. USA 2014, 111, 833-838. [CrossRef]

27. Tobia, M.J.; Yang, Q.X.; Karunanayaka, P. Intrinsic intranasal chemosensory brain networks shown by resting-state functional MRI. Neuroreport 2016, 27, 527-531. [CrossRef]

28. Gottfried, J.A.; Dolan, R.J. The nose smells what the eye sees: Crossmodal visual facilitation of human olfactory perception. Neuron 2003, 39, 375-386. [CrossRef]

29. Doty, R.L. Olfactory capacities in aging and Alzheimer's disease. Psychophysical and anatomic considerations. Ann. N. Y. Acad. Sci. 1991, 640, 20-27. [CrossRef]

30. Vasavada, M.M.; Wang, J.; Eslinger, P.J.; Gill, D.J.; Sun, X.; Karunanayaka, P.; Yang, Q.X. Olfactory cortex degeneration in Alzheimer's disease and mild cognitive impairment. J. Alzheimers Dis. 2015, 45, 947-958. [CrossRef]

31. Tabert, M.H.; Steffener, J.; Albers, M.W.; Kern, D.W.; Michael, M.; Tang, H.; Brown, T.R.; Devanand, D.P. Validation and optimization of statistical approaches for modeling odorant-induced fMRI signal changes in olfactory-related brain areas. Neuroimage 2007, 34, 1375-1390. [CrossRef] [PubMed]

32. Yaffe, K.; Freimer, D.; Chen, H.; Asao, K.; Rosso, A.; Rubin, S.; Tranah, G.; Cummings, S.; Simonsick, E. Olfaction and risk of dementia in a biracial cohort of older adults. Neurology 2017, 88, 456-462. [CrossRef] [PubMed]

33. Weiner, M.W.; Aisen, P.S.; Jack Jr, C.R.; Jagust, W.J.; Trojanowski, J.Q.; Shaw, L.; Saykin, A.J.; Morris, J.C.; Cairns, N.; Beckett, L.A.J.A.s.; et al. The Alzheimer's disease neuroimaging initiative: Progress report and future plans. Alzheimers Dement. 2010, 6, 202-211. [CrossRef] [PubMed] 
34. Yan, C.; Zang, Y. DPARSF: A MATLAB toolbox for "pipeline" data analysis of resting-state fMRI. Front. Syst. Neurosci. 2010, 4, 13. [CrossRef]

35. Yan, C.G.; Wang, X.D.; Zuo, X.N.; Zang, Y.F. DPABI: Data Processing \& Analysis for (Resting-State) Brain Imaging. Neuroinformatics 2016, 14, 339-351.

36. Anderson, J.S.; Druzgal, T.J.; Lopez-Larson, M.; Jeong, E.K.; Desai, K.; Yurgelun-Todd, D. Network anticorrelations, global regression, and phase-shifted soft tissue correction. Hum. Brain Mapp. 2011, 32, 919-934. [CrossRef]

37. Murphy, K.; Birn, R.M.; Handwerker, D.A.; Jones, T.B.; Bandettini, P.A. The impact of global signal regression on resting state correlations: Are anti-correlated networks introduced? Neuroimage 2009, 44, 893-905. [CrossRef]

38. Seubert, J.; Freiherr, J.; Djordjevic, J.; Lundstrom, J.N. Statistical localization of human olfactory cortex. Neuroimage 2013, 66, 333-342. [CrossRef]

39. Devanand, D.P.; Tabert, M.H.; Cuasay, K.; Manly, J.J.; Schupf, N.; Brickman, A.M.; Andrews, H.; Brown, T.R.; DeCarli, C.; Mayeux, R. Olfactory identification deficits and MCI in a multi-ethnic elderly community sample. Neurobiol. Aging 2010, 31, 1593-1600. [CrossRef]

40. Jack, C.R., Jr.; Knopman, D.S.; Jagust, W.J.; Shaw, L.M.; Aisen, P.S.; Weiner, M.W.; Petersen, R.C.; Trojanowski, J.Q. Hypothetical model of dynamic biomarkers of the Alzheimer's pathological cascade. Lancet Neurol. 2010, 9, 119-128. [CrossRef]

41. Hirano, A.; Zimmerman, H.M. Alzheimer's neurofibrillary changes: A topographic study. Arch. Neurol. 1962, 7, 227-242. [CrossRef] [PubMed]

42. De Lacoste, M.C.; White, C.L., 3rd. The role of cortical connectivity in Alzheimer's disease pathogenesis: A review and model system. Neurobiol. Aging 1993, 14, 1-16. [CrossRef]

43. Brier, M.R.; Thomas, J.B.; Ances, B.M. Network dysfunction in Alzheimer's disease: Refining the disconnection hypothesis. Brain Connect. 2014, 4, 299-311. [CrossRef] [PubMed]

44. Braak, H.; Braak, E. Morphological criteria for the recognition of Alzheimer's disease and the distribution pattern of cortical changes related to this disorder. Neurobiol. Aging 1994, 15, 355-356. [CrossRef]

45. Braak, H.; Braak, E. Frequency of stages of Alzheimer-related lesions in different age categories. Neurobiol. Aging 1997, 18, 351-357. [CrossRef]

46. Braak, H.; Braak, E. The human entorhinal cortex: Normal morphology and lamina-specific pathology in various diseases. Neurosci. Res. 1992, 15, 6-31. [CrossRef]

47. Hyman, B.T.; Van Hoesen, G.W.; Damasio, A.R.; Barnes, C.L. Alzheimer's disease: Cell-specific pathology isolates the hippocampal formation. Science 1984, 225, 1168-1170. [CrossRef]

48. Hyman, B.T.; Van Hoesen, G.W.; Kromer, L.J.; Damasio, A.R. Perforant pathway changes and the memory impairment of Alzheimer's disease. Ann. Neurol. 1986, 20, 472-481. [CrossRef]

49. Delbeuck, X.; van der Linden, M.; Collette, F. Alzheimer's disease as a disconnection syndrome? Neuropsychol. Rev. 2003, 13, 79-92. [CrossRef]

50. Zuo, X.N.; Ehmke, R.; Mennes, M.; Imperati, D.; Castellanos, F.X.; Sporns, O.; Milham, M.P. Network centrality in the human functional connectome. Cereb. Cortex 2012, 22, 1862-1875. [CrossRef]

51. Lacalle-Aurioles, M.; Navas-Sanchez, F.J.; Aleman-Gomez, Y.; Olazaran, J.; Guzman-De-Villoria, J.A.; Cruz-Orduna, I.; Mateos-Perez, J.M.; Desco, M. The Disconnection Hypothesis in Alzheimer's Disease Studied Through Multimodal Magnetic Resonance Imaging: Structural, Perfusion, and Diffusion Tensor Imaging. J. Alzheimers Dis. 2016, 50, 1051-1064. [CrossRef]

52. Allen, G.; Barnard, H.; McColl, R.; Hester, A.L.; Fields, J.A.; Weiner, M.F.; Ringe, W.K.; Lipton, A.M.; Brooker, M.; McDonald, E.; et al. Reduced hippocampal functional connectivity in Alzheimer disease. Arch. Neurol. 2007, 64, 1482-1487. [CrossRef]

53. Zhang, H.Y.; Wang, S.J.; Xing, J.; Liu, B.; Ma, Z.L.; Yang, M.; Zhang, Z.J.; Teng, G.J. Detection of PCC functional connectivity characteristics in resting-state fMRI in mild Alzheimer's disease. Behav. Brain Res. 2009, 197, 103-108. [CrossRef] [PubMed]

54. Karunanayaka, P.R.; Wilson, D.A.; Tobia, M.J.; Martinez, B.E.; Meadowcroft, M.D.; Eslinger, P.J.; Yang, Q.X. Default mode network deactivation during odor-Visual association. Hum. Brain Mapp. 2017, 38, 1125-1139. [CrossRef] [PubMed] 
55. Lu, J.; Yang, Q.X.; Zhang, H.; Eslinger, P.J.; Zhang, X.; Wu, S.; Zhang, B.; Zhu, B.; Karunanayaka, P.R. Disruptions of the olfactory and default mode networks in Alzheimer's disease. Brain Behav. 2019, 9, e01296. [CrossRef]

56. Filippi, M.; Basaia, S.; Canu, E.; Imperiale, F.; Meani, A.; Caso, F.; Magnani, G.; Falautano, M.; Comi, G.; Falini, A.; et al. Brain network connectivity differs in early-onset neurodegenerative dementia. Neurology 2017, 89, 1764-1772. [CrossRef]

57. Hamalainen, A.; Pihlajamaki, M.; Tanila, H.; Hanninen, T.; Niskanen, E.; Tervo, S.; Karjalainen, P.A.; Vanninen, R.L.; Soininen, H. Increased fMRI responses during encoding in mild cognitive impairment. Neurobiol. Aging 2007, 28, 1889-1903. [CrossRef]

58. Heun, R.; Freymann, K.; Erb, M.; Leube, D.T.; Jessen, F.; Kircher, T.T.; Grodd, W. Mild cognitive impairment (MCI) and actual retrieval performance affect cerebral activation in the elderly. Neurobiol. Aging 2007, 28, 404-413. [CrossRef]

59. Dickerson, B.C.; Sperling, R.A. Large-scale functional brain network abnormalities in Alzheimer's disease: Insights from functional neuroimaging. Behav. Neurol. 2009, 21, 63-75. [CrossRef]

60. Machulda, M.M.; Ward, H.A.; Borowski, B.; Gunter, J.L.; Cha, R.H.; O’Brien, P.C.; Petersen, R.C.; Boeve, B.F.; Knopman, D.; Tang-Wai, D.F.; et al. Comparison of memory fMRI response among normal, MCI, and Alzheimer's patients. Neurology 2003, 61, 500-506. [CrossRef]

61. Bakker, A.; Krauss, G.L.; Albert, M.S.; Speck, C.L.; Jones, L.R.; Stark, C.E.; Yassa, M.A.; Bassett, S.S.; Shelton, A.L.; Gallagher, M. Reduction of hippocampal hyperactivity improves cognition in amnestic mild cognitive impairment. Neuron 2012, 74, 467-474. [CrossRef] [PubMed]

62. Zott, B.; Simon, M.M.; Hong, W.; Unger, F.; Chen-Engerer, H.J.; Frosch, M.P.; Sakmann, B.; Walsh, D.M.; Konnerth, A. A vicious cycle of beta amyloid-dependent neuronal hyperactivation. Science 2019, 365, 559-565. [CrossRef] [PubMed]

63. Postuma, R.B.; Gagnon, J.F.; Vendette, M.; Desjardins, C.; Montplaisir, J.Y. Olfaction and color vision identify impending neurodegeneration in rapid eye movement sleep behavior disorder. Ann. Neurol. 2011, 69, 811-818. [CrossRef] [PubMed]

64. Power, J.D.; Barnes, K.A.; Snyder, A.Z.; Schlaggar, B.L.; Petersen, S.E. Spurious but systematic correlations in functional connectivity MRI networks arise from subject motion. Neuroimage 2012, 59, 2142-2154. [CrossRef] [PubMed]

65. Zhang, J.; Hao, C.; Jiang, J.; Feng, Y.; Chen, X.; Zheng, Y.; Liu, J.; Zhang, Z.; Long, C.; Yang, L. The mechanisms underlying olfactory deficits in apolipoprotein E-deficient mice: Focus on olfactory epithelium and olfactory bulb. Neurobiol. Aging 2018, 62, 20-33. [CrossRef] [PubMed]

66. Petersen, R.C. Mild cognitive impairment. Lancet 2006, 367, 1979. [CrossRef]

67. Lafaille-Magnan, M.E.; Poirier, J.; Etienne, P.; Tremblay-Mercier, J.; Frenette, J.; Rosa-Neto, P.; Breitner, J.C.S. Odor identification as a biomarker of preclinical AD in older adults at risk. Neurology 2017, 89, 327-335. [CrossRef]

(C) 2019 by the authors. Licensee MDPI, Basel, Switzerland. This article is an open access article distributed under the terms and conditions of the Creative Commons Attribution (CC BY) license (http://creativecommons.org/licenses/by/4.0/). 